\title{
AMPUTATIONS IN DIABETICS
}

\author{
By R. C. F. Catterall, M.Chir., F.R.C.S. \\ Orthopaedic Consultant, King's College Hospital
}

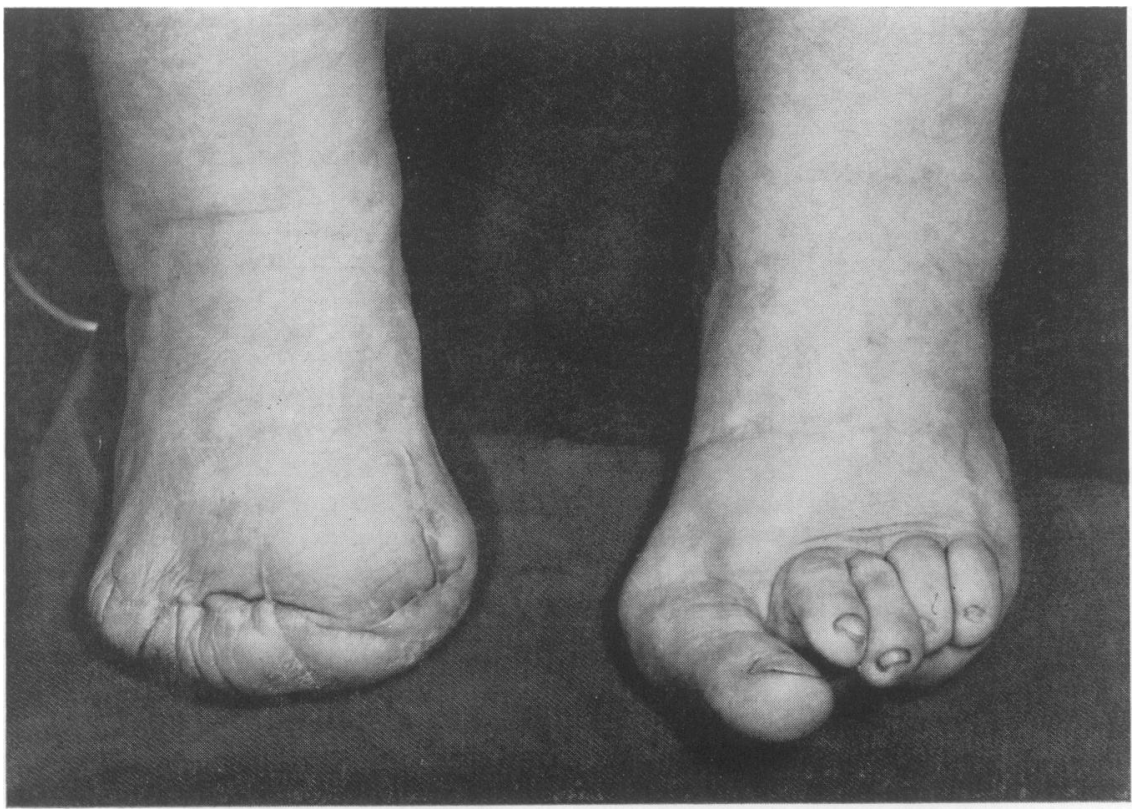

Fig. 1. - Neuropathic deformity (left). Five-toe-ectomy (right).

It is generally agreed that diabetics, more frequently than other patients, develop lesions on their feet which lead them to submit to amputation of the leg either as a life-saving necessity or as an escape from intolerable pain. It is the object of this paper to show that a fuller understanding of the cause of these lesions, together with a régime of treatment based on this knowledge, will allow less mutilating operations to be successfully accomplished and may on occasions obviate the necessity of amputation.

The existence of so-called diabetic gangrene has been known for a very long time. Sir James Paget was aware of two types of gangrene; a dry painful one which did badly, and a warmer and less painful type which often did well. $\mathrm{He}$ was opposed to operative intervention on account of the mortality, and pursued a policy of masterly inactivity characterized by substantial doses of opium and the use of deodorant unguents. With an improvement in anaesthesia radical surgery was more readily undertaken, but it was found that bad results followed any amputation below the thigh unless the problem could be solved by removing only one toe. The post-operative sloughing was ascribed to the raised blood-sugar which was alleged to militate against healing and form an ideal culture medium for any secondarily infecting organisms.

The aetiological factors leading to the various lesions have been defined by Oakley as:
(a) Sepsis.
(b) Neuropathy.
(c) Ischaemia.
(d) Combinations of (a), (b) and (c).

Uncomplicated sepsis in an otherwise normal foot is very uncommon and rarely leads to amputation. The combined form is the most frequently 
encountered but the dominant factor can always be established and will dictate the régime of treatment to be followed. It is therefore prudent to consider the clinical manifestations of neuropathy and ischaemia in some detail.

\section{Clinical Signs of Neuropathy}

The insidious degeneration of myelinated and non-myelinated nerve fibres, associated less with the duration than with the control of the diabetic state, results in well marked sensory and motor signs. The fully developed state with a foot drop or Charcots Arthropathy is rare, but lesser degrees of the condition are often encountered. The limb will then show the following changes:

\section{(I) Sensory}

Paraesthesiae in the lower limbs are followed by impairment of the pain sense which often precedes loss of touch. The ankle jerks are frequently diminished or lost, as is vibration sense, and trophic changes in the skin render it simultaneously brittle and difficult to cleanse. Interference with the autonomic motor response assists in lowering the local tissue to trauma.

\section{(2) Motor}

Loss of function in the intrinsic muscles of the foot results in a characteristic deformity of the toes (Fig. I). These become subluxed and clawed, and are soon splinted in this position by a combination of disuse and minimal sepsis. The deformed position prevents the normal movements of walking and places an undue strain on the devitalized skin beneath the metatarsal heads. The resulting callosities are easily converted into ulcers be amateur chiropody and a secondary infection from a shoe rub completes the picture of disaster.

It is to be emphasized that these severely neuropathic feet are warm to the touch and the skin of non weight bearing areas remains relatively elastic although characteristically hyperkeratotic. The pulses can usually be felt without difficulty and any gangrene that develops usually follows superadded septic thrombosis.

\section{Clinical Signs of Ischaemia}

With the exception of the relatively rare calamity following the sudden obstruction of a major vessel the onset of ischaemic symptoms is usually gradual. A feeling of coldness in the limb is accompanied by intermittent claudication of the calf. The foot later becomes the seat of a burning pain, which is characteristically made worse by heat and therefore worse at night. The consequent loss of sleep is a serious complication. The whole limb develops a

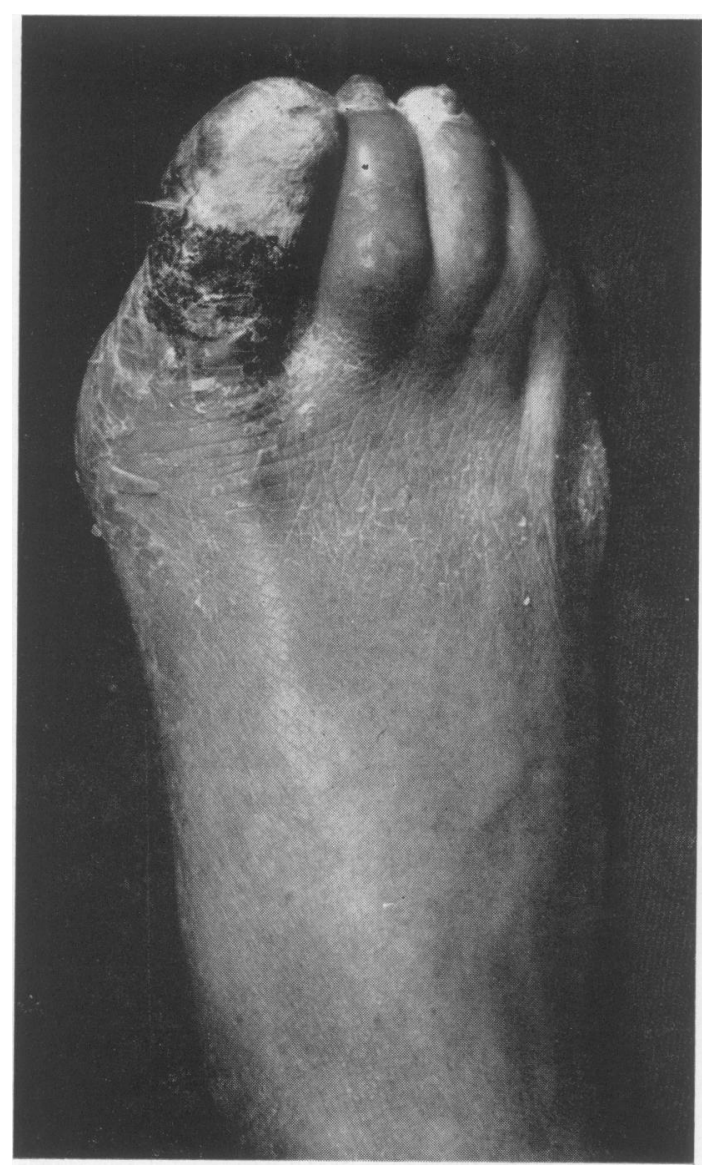

FIG. 2,-Ischaemic foot.

shrivelled dry appearance with shiny hairless skin which may be either pale or salmon pink in colour (Fig. 2). The painful foot is characteristically pink.

Ultimately frank gangrene develops either distally in one or more toes or else in the skin of the heel. This latter and sinister conplication is often precipitated by decubitus and may be prevented by suspension of the limb.

\section{Septic and Combined Lesions}

The addition of sepsis will change the clinical picture for the worse. In the neuropathic foot the blood supply will allow a normal inflammatory reaction to occur, although the absence of pain will often delay the patient's complaint until the septic condition is well advanced. A septic arthritis is not infrequently the presenting symptom. In the ischaemic foot, however, sepsis produces a rapidly spreading gangrene. 

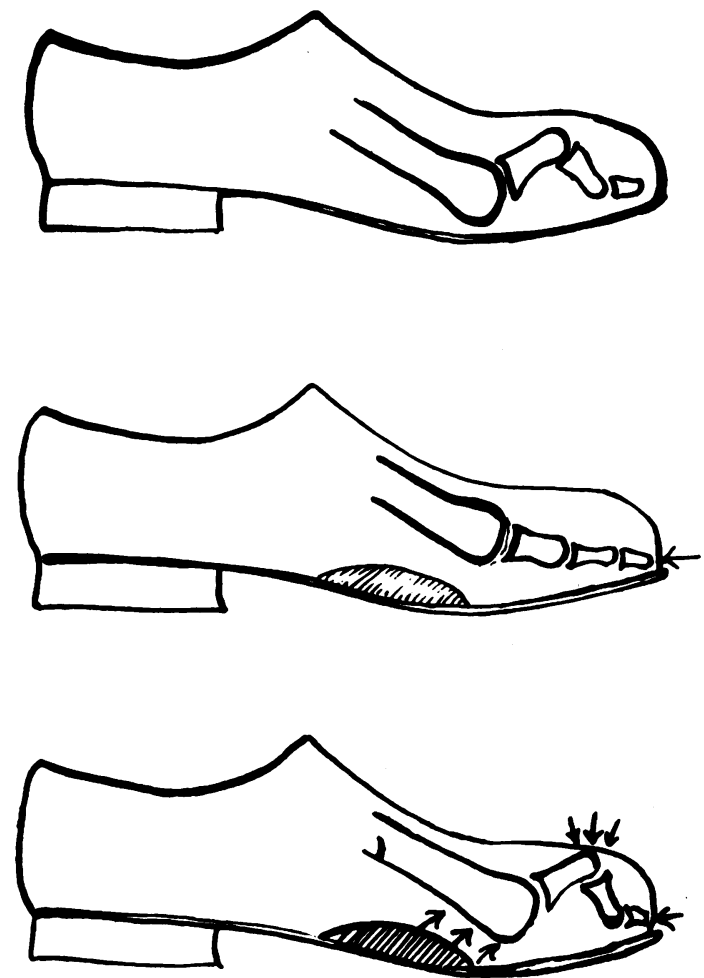

FIG. 3.-Showing how appliances inside the shoe will produce new sites of pressure, especially when the toes are stiff.

\section{Treatment}

Prophylactic - the vast majority of the lesions which lead to amputation stem from an abrasion consequent on a shoe-rub. The most important single feature in prophylaxis is the provision of shoes of adequate size with soft and pliable uppers. Prudent padding of prominent bony points is often advisable, but in applying it great care must be taken to ensure that other parts of the feet are not subjected to shoe pressure. It cannot be overemphasized that the use of space-occupying appliances inside the shoe will only precipitate the very disasters it is hoped to prevent (Fig. 3). The routine cleansing and softening of the skin with soap, water and lanoline is also of great importance. In the event of the patient being confined to bed for any length of time the heels must be prevented from pressing against the mattress. We have found that suspension by the method shown in Fig. 4 is the easiest method of ensuring this. The slings are made of canvas and well padded by felt. The suspension from an overhead beam is by standard Terry springs adjusted to lift the heel an inch or two clear of the mattress.

\section{Surgical}

General Principles. Irrespective of whether the lesion is predominately ischaemic or neuropathic, certain basic principles of surgical treatment can be laid down.

I. The condition of the feet rarely carries an urgent risk to life, and snap decisions about amputation are to be deprecated. In almost every case a short period of rest with the leg elevated, combined with adequate doses of antibiotics and rigid control of the diabetes with two daily doses of soluble insulin, will usually produce improvement and may allow less radical surgery to be advised.

2. Expert general anaesthesia is essential. These patients are always poor risks and may be a very difficult anaesthetic problem. We have had no experience of freezing but feel that the results of inhalation anaesthesia are so good that experimenting with a method which may further impair the viability of the devitalized flaps is not justified.

3. No sort of tourniquet must ever be used on any of the limbs at any level.

4. The sound limb must be supported on a soft foam pillow throughout the whole period of narcosis. The risk of pressure necrosis of the sound heel is everpresent, and neglect of this precaution may cost the patient his hitherto 'good' foot.

5. No sort of tension must be permitted to build up in the wound at any stage of the operation or post-operative treatment. Such tension, often attributable to well meaning but ambitious attempts to obtain a primary cosmetic result, is the precipitating factor in the post-operative spread of gangrene. The writer is well aware of many successes which have followed primary suture; the failures of this method have to submit to two major amputations within a few days: not all of them survive the second, avoidable, operation. The apposition of skin edges lying in place without tension can often be aided by an encircling bandage of well-vaselined gauze. If this is then split on the opposite side of the foot no constrictive effect is possible yet some security of the line of incision is still maintained.

6. In every foot needing operative treatment it will be found that the tissues are desensitized by neuropathy and divitalized by ischaemia to a greater or less degree. It cannot be denied that the design of any amputation is of necessity somewhat barbaric and crude, but this does not excuse the exercise of gentleness in its performance. Time spent in gentle handling of the tissues at operation is well repayed by the shortening of the convalescence. 


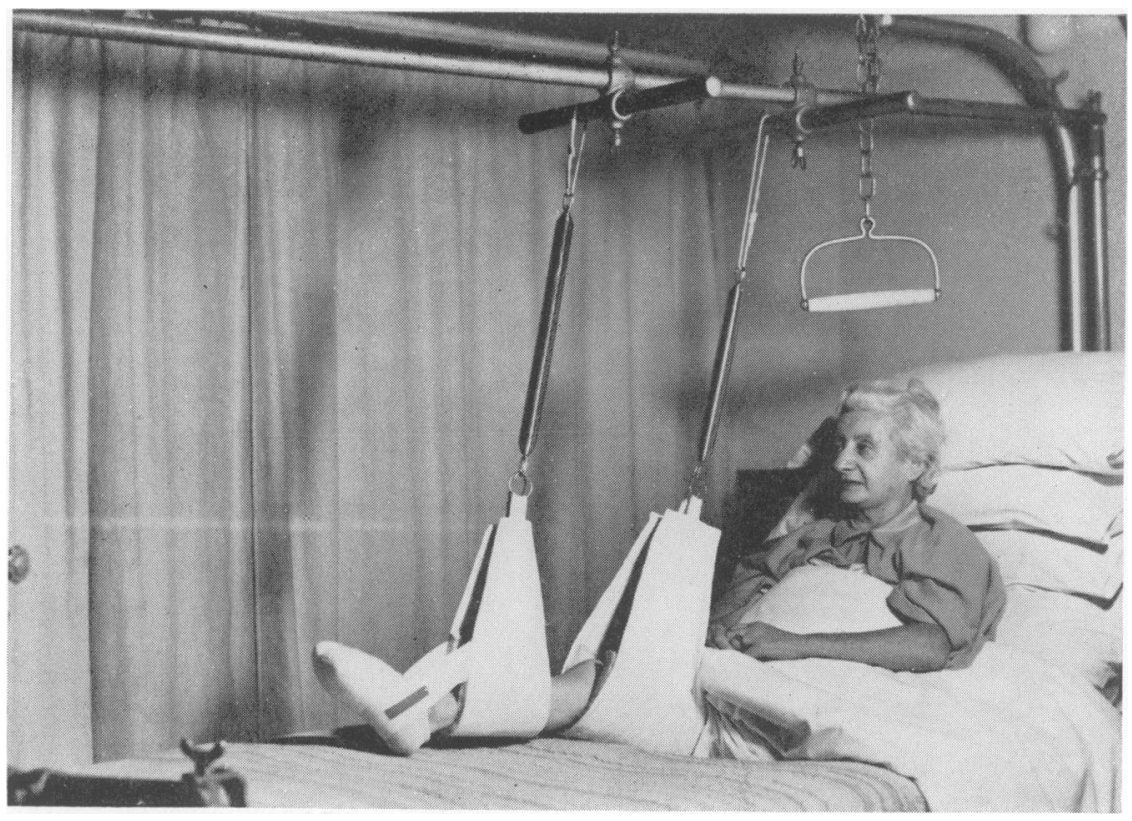

Fig. 4.-Suspension apparatus as used at King's College Hospital.

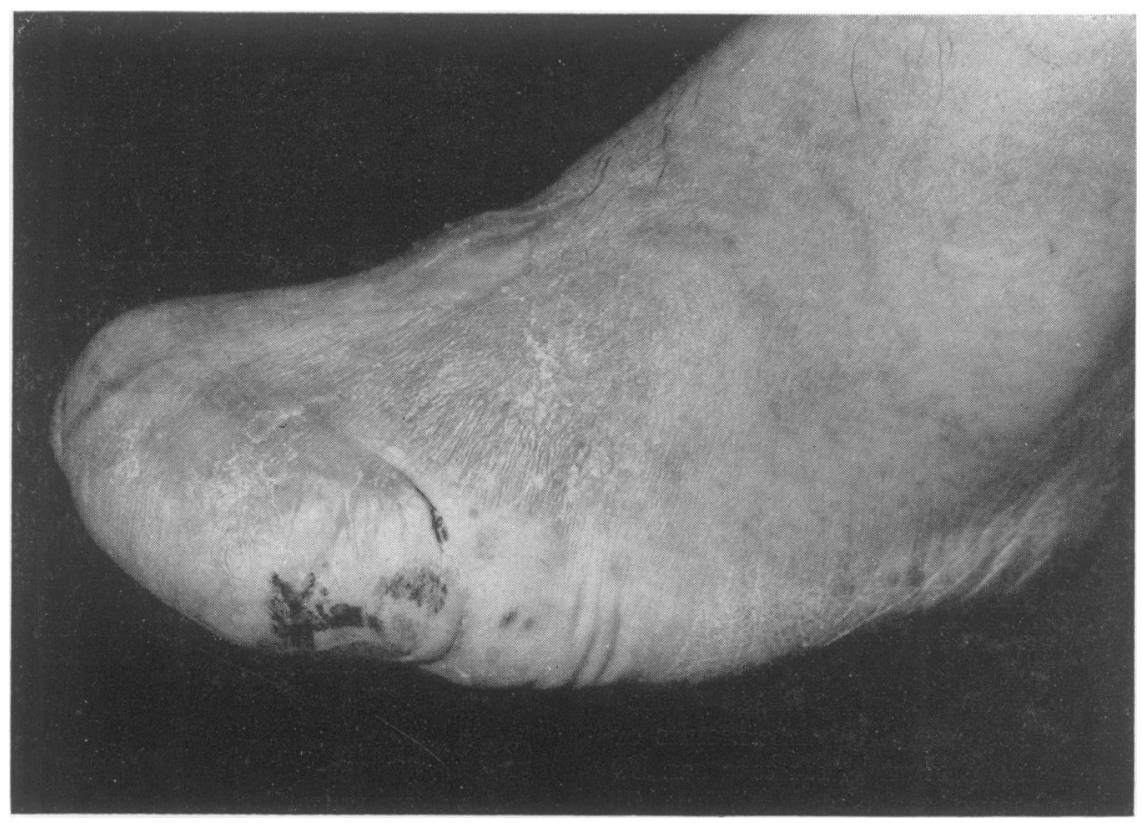

Neuropathic

In the essentially neuropathic foot the blood supply will be adequate for healing and the individual lesion may be dealt with on its own merits. In the absence of gross infection the cause of the lesion is always a pathologically

FIG. 5.-Result of five-toe-ectomy. 


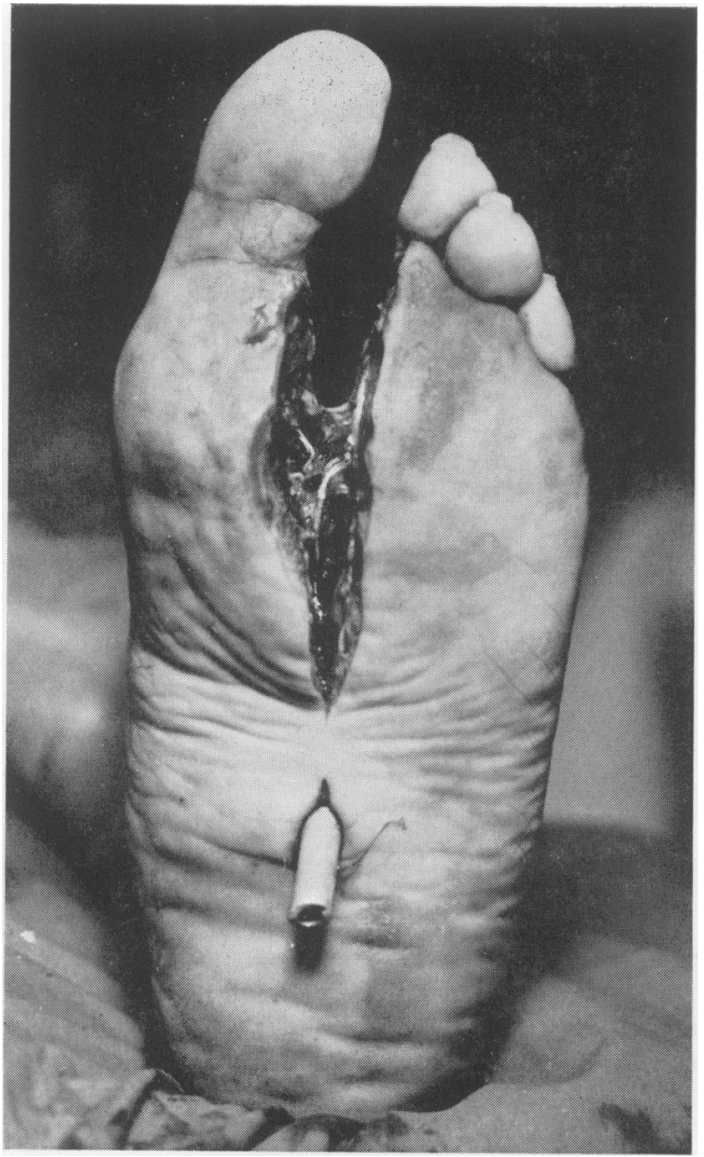

Fig. 6a.-Incision for drainage of abscess in second toe flexor tendon.

prominent bony protuberance. The surgical adjustment necessary consists in removing sufficient bone to allow the surrounding skin to fall together without tension. This often involves sacrificing a toe and part or whole of the appropriate metatarsal. On the lateral side of the foot even heroic bone section will leave thin flaps stretched to their limit. Under these circumstances primary Thiersch grafting may be employed with every prospect of success. In planning these procedures thought must be given to the late results of the amputations. The first and fifth toes can be sacrificed by Faraboeufs technique without ill effect, but removal of more than one of the middle three toes will always be followed by deviation of the other toes into the gap. This will produce new sites of shoe pressure and demand further surgery. Removal of all five toes through dorsal incisions will produce a far more shapely foot (Fig. 5). We have no experience of planned

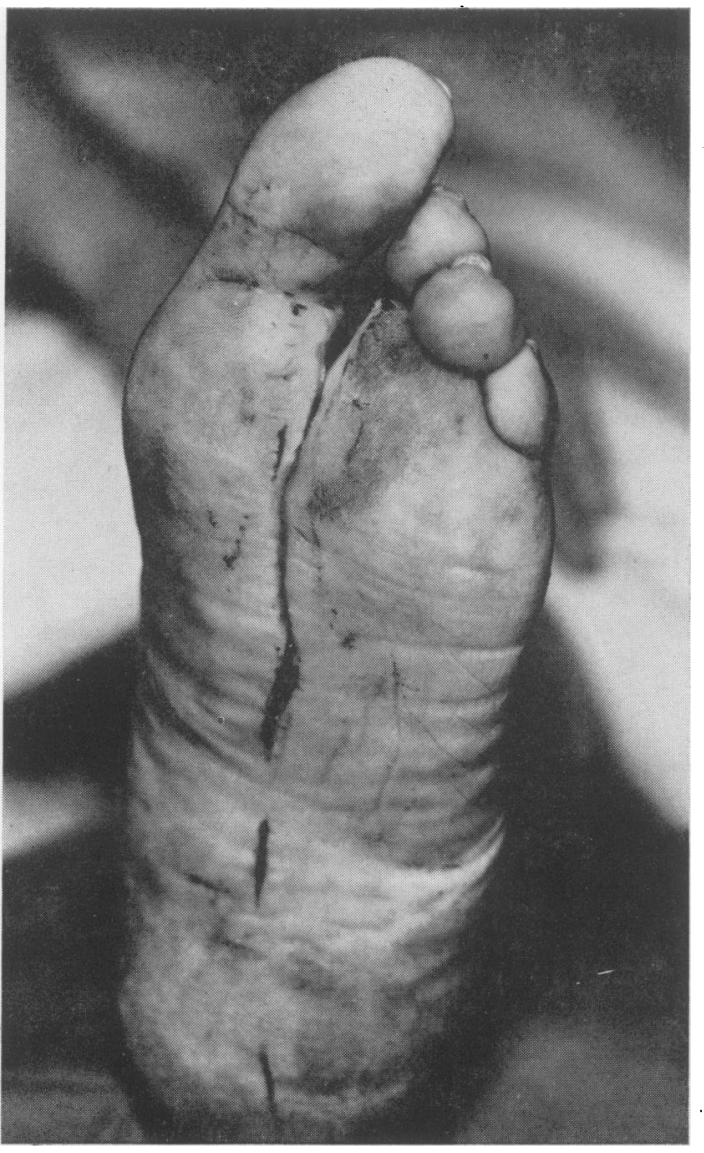

Fig. 6b.-Same case 14 days later.

transmetatarsal amputations but feel that patients whose circulation cannot heal up a 'five-toeectomy' will need a below-knee amputation. We also remain unconvinced of the value of the Chopard and Piragoff amputation for this condition, since the skin flaps will have to be sutured and will very probably necrose. Where the midtarsal joint is disorganized a below-knee amputation is essential.

\section{Septic Neuropathic}

As previously stated the loss of pain sense will often allow quite large abscesses to form before the patient seeks advice. The abscesses are to be drained through free incisions so designed as to divert the gravitational flow of pus to the surface. Bone should be sacrificed fearlessly in draining these abscesses, preferably by disarticulation to avoid exposing raw bone to contamination by pus. 
Once the infection is controlled healing is rapid provided that the skin edges can fall together without tension (Fig. 6a and b).

\section{Ischaemic}

In these feet the whole surgical plan is conditioned by the blood supply and hampered by the inelasticity of the skin. The blood supply has already proved itself insufficient to maintain viability in the toes, and cannot therefore be expected to accommodate itself to the hyperaemia required for rapid healing. In particular the filling in of dead space by granulation will rarely occur in these feet, and skin grafts will never ' take.'

Individual dry gangrene of the toes is one of the most frequently presented problems. The risk of amputation is the production of an indolent ulcer over the metatarsal head, and the occasional occurrence of spontaneous separation and healing may encourage a policy of masterly inactivity. In our experience such a policy carried too high a risk of sudden and calamitous spread after trauma or infection and all these toes should be amputated as a matter of urgency. Sufficient bone should be removed to allow the skin edges to fall together, but no sutures should be used. After amputation the foot should be kept cool and elevated until healing is well advanced. If after Io to I4 days a static stage has been reached a trial period of walking should be advised. In some cases the activity will produce sufficient improvement in the circulation to allow healing to proceed: in others the additional load will precipitate the spread of gangrene and render the ultimately inevitable amputation imperative. Forcing the decision in this way not only spares the patient anxiety but also cuts short unnecessary pain which, if long continued, is often felt in the phantom for many weeks.

The more advanced degrees of ischaemia or neuropathy will produce a foot which is a burden to its owner on account of pain or disorganization of the major joints. The addition of sepsis may produce an urgent danger to life and render amputation a matter of urgency. In all such cases, irrespective of clinical evidence of gross impairment of permeability of the popliteal artery, a below-knee amputation will be successful if carried out by the method described by Silbert. ${ }^{3}$ A circular incision is made through skin and deep fascia 8 in. below the knee. The skin and deep fascia are gently reflected upwards for 2 in. and the muscles and nerves divided at this level. The dissection is then continued in the subperiosteal layer for a further 2 in. up the tibia and a little further up the fibula. The bones are then sawn through, care being taken to bevel the tibia in such a way as to avoid pressure on the skin by the antero-medial angle. After control of any unsecured bleeding points in soleus or gastrocnemius the soft tissues are allowed to fall down over the bone are not secured in any way. The stump at this stage is most unsightly, but all temptation to tidy it up must be vigorously resisted. A dressing of vaselined-gauze and wool is applied and the whole stump supported in a trough of plaster of paris. On the tenth day the patient is again anaesthetized and the wound inspected. If all is well a delayed primary suture may be carried out, but any degree of infection however slight makes this procedure most inadvisable and may produce extensive sloughing of the flaps. After redressing, the stump is supported in the gutter until healing is practically complete, to prevent a flexion deformity of the knee. Increasing experience of this method of amputation leads to delayed primary suture being used less frequently. All the stumps are soundly healed in six to eight weeks without it, and limb-filling may be commenced in four months or less. This method has been in use at the diabetic department of King's College Hospital for the past three years and in the first 100 amputations there was no case of failure to heal, although three needed a minor sequestrectomy at some stage in the healing, and not a single death attributable to the operation. I am completely convinced that this technique is a major advance in the surgery of these patients, and, by conserving the knee, has greatly eased their lot. Even double amputatees can often manage to live a wheel-chair life in their own homes.

The problem of amputation in diabetics is not yet solved, but an increasing understanding of the aetiological factors involved has allowed the operations to be more accurately planned, and thereby vouchsafed a greater proportion of success. More intensive prophylactic treatment based on lines described above will make the indications for amputation less frequent, and the operations less mutilating. It is to be hoped that with further study the outlook for an elderly diabetic with a lesion on the foot, though never rosy, will seldom become desperate. 